\title{
Misoprostol (Cytotec) for prevention of ovarian hyperstimulation syndrome in cases of in vitro fertilization
}

Leonardo Formigli ${ }^{1}$, Graziella Badulli ${ }^{1 *}$, Ramarolahy Rija ${ }^{2}$, Marcienne Aimée ${ }^{1}$, Ramachandran Krishnamoorthy ${ }^{1}$ Hanitra Rasoarimanana $^{1}$ and Rakotobe Andriamaro ${ }^{1}$

${ }^{1}$ CE-FER-MAD (Centre de Fertilité de Madagascar), Antananarivo, Madagascar

${ }^{2}$ Service de Réanimation Chirurgicale (Hôpital Universitaire Ravoahangy), Antananarivo, Madagascar

\section{Letter to Editor}

We describe here a possible new treatment for the prevention or reduction of ovarian hyperstimulation syndrome (OHSS), when using the In Vitro Fertilization (IVF) technique. This syndrome may occur, with various degrees of severity leading even to life threatening situations, in patients being administered ovarian stimulation drugs for assisted reproductive procedures, with the aim to obtain many oocytes and increase the chance of success [1].

In IVF, with the advent of the very successful vitrification procedure [2], it is now possible to avoid late OHSS by freezing all embryos and postponing Embryo Transfer (ET) to a subsequent cycle. In fact, late OHSS may only occur if ET is performed during the IVF cycle, in patients getting pregnant. However OHSS may still occur during the two luteal weeks between Oocyte Pick Up (OPU) and the menses.

The cystic enlargement of the ovaries and particularly the high number of corpora lutea which develop during these stimulated cycles are thought to be responsible for the release of vasoactive substances such as the Vascular Endothelial Growth Factor (VEGF) which are implicated in increasing vascular permeability [3]. This causes a massive fluid shift from the intravascular bed to the third compartment, giving rise to the OHSS.

The usual treatment of the syndrome is symptomatic: i.v. albumin to replace the albumin lost in the third space with the aim to increase the plasma onchotic pressure; administration of heparin to avoid thromboembolism due to the haemoconcentration and i.v. fluids to reduce haemoconcentration. Cabergoline [4] has been found to partially inhibit the VEGF receptor 2 phosphorilation levels and associated vascular permeability: the efficacy of this drug seems to be higher in mild cases of OHSS.

Misoprostol is a prostaglandin analogue used for the treatment of peptic ulcer; it is also widely used off label since decades for starting labour, just prior to uterine dilation and curettage, before hysteroscopy and as an abortifacient. Prostaglandins are also used in cattle breeding as luteolytic agents for synchronizing cows' cycles for artificial reproduction [5].

Three years ago we thought to take advantage of this luteolytic property of prostaglandins in order to induce luteolysis in patients undergoing IVF, where it was decided to vitrify all embryos, since their ovaries were heavily stimulated and there was a risk of OHSS.
We submitted the protocol to our Ethic Committee and obtained its approval.

All patients undergoing OPU came back to the Centre 2 days later and were checked by ultrasound and clinical examination. If there was any sign or symptom that OHSS may have developped such as stomach-ache, abdominal pain, nausea or vomiting, breathing difficulties, presence of free fluid - more than $3 \mathrm{~cm}$ vertically - in the Douglas pouch, ovarian diameter greater than $7 \mathrm{~cm}$, pleural effusion, it was decided to freeze all embryos, cancel the Embryo Transfer and administer Misoprostol (prostaglandin E1, tablets - Cytotec, Pfizer), $800 \mathrm{mcg}$ in a single vaginal administration that same day. All these patients signed an informed consent form.

We observed that almost all patients had considerably improved subjectively or objectively, starting from the day after Cytotec administration. A few patients however did not improve and we administered a second dose 48 hours after the first one and they also recovered soon. What impressed us was the short delay between Misoprostol administration and the improvement of the patients' conditions. It is tempting to speculate that Misoprostol administered vaginally reaches the ovaries through a 'first pass' and promptly induces an at least partial luteolytic effect thus stopping the chain of events leading to a full blown OHSS.

After the first 10 cases, all successful, the team asked the Ethic Committee to promote a prospective randomized control study, but it was decided not, since it was unethical to avoid administering Cytotec to part of the patients. In the last 3 years, Cytotec was administered to 113 cases out of 679 OPUs and none needed any hospital admission, paracenthesis, thoracenthesis, fluid i.v. replacement or any other remedy against OHSS, whereas in the past, the rate of complications due to OHSS was $5-6 \%$ of the OPUs.

A particularly interesting application of this OHSS preventive strategy is for oocyte donors, who undergo ovarian stimulation and OPU, but are not transferred any embryo. Misoprostol should not be used for infertile patients undergoing other assisted reproductive techniques such as ovarian stimulation coupled with natural intercourse, insemination, Gamete Intra Fallopian Transfer (GIFT) and

${ }^{\star}$ Correspondence to: Graziella Badulli, CE-FER-MAD (Centre de Fertilité de Madagascar), Antananarivo, Madagascar, E-mail: graziellaparda@yahoo.it

Received: February 01, 2018; Accepted: February 08, 2019; Published: February 11,2019 
similar techniques, since Misoprostol may induce an abortion in such cases.

In conclusion, intravaginal administration of Cytotec coupled with "all embryos" vitrification may avoid OHSS during the luteal phase of IVF cycles. Cytotec should be used only with in vitro techniques, where embryos are produced extracorporeally. It is important to stress that no embryo should be transferred during those IVF cycles and ETs should be postponed to subsequent cycles. Oocyte donors are very suitable for this OHSS preventive strategy since they undergo ovarian stimulation and OPU, but not ET.

Misoprostol should not be used with in vivo assisted reproduction techniques, whenever fertilization takes place intracorporeally, since Misoprostol is a powerful abortifacient.

\section{Financial support}

There was no financial support or any relationships that may lead to a conflict of interest.

\section{Conflict of interest}

There was no conflict of interest whatsoever.

\section{References}

1. Delvigne A and Rozenberg S (2002) Epidemiology and prevention of ovarian hyperstimulation syndrome (OHSS): a review. Hum Reprod Update 8: 559-577. [Crossref]

2. Valojerdi MR, Eftekhari-Yazdi P, Karimian L, Hassani F, Movaghar B (2009) Vitrification versus slow freezing gives excellent survival post warming embryo morphology and pregnancy outcomes for human cleaved embryos. J Assist Reprod Genet 26: 347-354. [Crossref]

3. Kumar P, Sait S, Sharma A, Kumar M (2011) Ovarian hyperstimulation syndrome. $J$ Hum Reprod Sci 4: 70-75. [Crossref]

4. Humaidan P, Quartarolo J and Papanikolau E (2010) Preventing ovarian hyperstimulation syndrome: guidance for the clinician. Fertil Steril 94: 389-400. [Crossref]

5. Archibald L T, Tran T, Massey R, Klapsfein E (1992) Conception rates in dairy cows after timed-insemination and simultaneous treatment with gonadotropin-releasing hormone and/or prostaglandin F2 $\alpha$. Theriogenology 37: 723-726.

Copyright: (C2019 Formigli L. This is an open-access article distributed under the terms of the Creative Commons Attribution License, which permits unrestricted use, distribution, and reproduction in any medium, provided the original author and source are credited. 